\title{
Intra-cavity patterning - a new method of single mode emission enhancement
}

\author{
Tomasz Czyszanowski, ${ }^{* 1}$ Nicolas Volet, ${ }^{2}$ Jarosław Walczak, ${ }^{1}$ Maciej Dems, ${ }^{1}$ Robert Sarzała ${ }^{1}$ \\ ${ }^{1}$ Photonics Group, Institute of Physics, Lodz University of Technology, ul. Wolczanska 219, 90-924 Lodz, Poland, \\ ${ }^{2}$ Laboratory of Physics of Nanostructures, Ecole Polytechnique Federale de Lausanne (EPFL), 1015 Lausanne, \\ Switzerland
}

Received August 21, 2013; accepted February 11, 2014; published March 31, 2014

\begin{abstract}
We demonstrate the results of numerical simulations of $1.3 \mu \mathrm{m} \mathrm{InAlGaAs/InP} \mathrm{VCSEL} \mathrm{with} \mathrm{a} \mathrm{ring} \mathrm{pattern} \mathrm{etched} \mathrm{at} \mathrm{the} \mathrm{interface}$ between the cavity and top DBR. We show that the proposed design offers selective confinement of the fundamental mode and strong discrimination of higher order modes. Consequently, VCSEL with ring confinement is capable to operate in a broad range of injected currents in the single mode regime, which facilitates the improvement of maximal emitted power and increase of the wavelength tuning range with very narrow spectral characteristics.
\end{abstract}

High power, single-mode emitting vertical-cavity surface-emitting lasers (VCSELs) are of great interest, since they can serve as high-power, smoothly tuned with current and temperature, coherent-optical sources. Emerging applications include inexpensive and portable gas sensors, telecommunication emitters, and so on. For these applications, it is preferable to have a low-threshold, high side-mode suppression ratio (SMSR) light source. VCSELs inherently emit in a single longitudinal mode; controlling the lateral mode is far more complicated. It can be achieved using a well-established wet oxidation technology, which makes possible single-mode operation only for relatively narrow apertures (providing less than 5 $\mathrm{mW}$ of output power) [1]. Other methods provide up to twofold higher emitted power but exploit more challenging technologies [2-5]. The limitation of high power single mode operation comes from small optical apertures or multimode operation under high injection levels. This paper provides numerical analysis of the ring pattern etched in the cavity of the VCSEL which eliminates higher order mode emission. The ring pattern provides confinement for a fundamental mode and forces higher order modes to be pushed out of the active region.

The analyzed structure (Fig. 1a) [6] incorporates InAlGaAs quantum wells within an InP cavity. The cavity is bounded by 35 pairs of $\mathrm{Al}_{0.9} \mathrm{Ga}_{0.1} \mathrm{As} / \mathrm{GaAs} \mathrm{DBR}$ from the bottom and 20.5 pairs of $\mathrm{Al}_{0.9} \mathrm{Ga}_{0.1} \mathrm{As} / \mathrm{GaAs}$ DBRs from the top. Laterally patterned tunnel junction (TJ) layers are responsible for tunnelling carriers into the

\footnotetext{
*E-mail: tomasz.czyszanowski@p.lodz.pl
}

active region. In the analysis, we consider a circular shaped TJ mesa of a diameter varying from 6 to $8 \mu \mathrm{m}$.

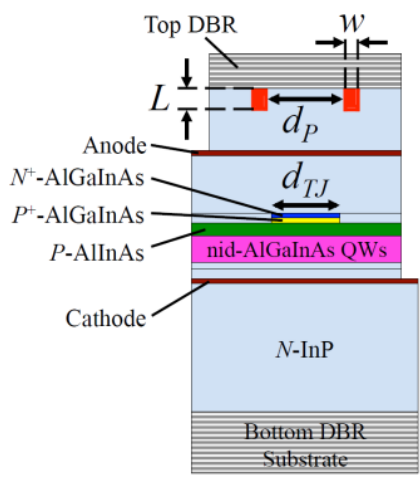

a)

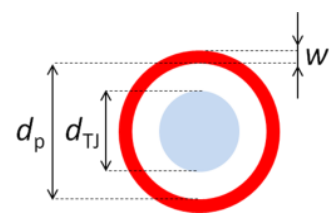

b)
Fig. 1. a) Schematic cross-section of the patterned VCSEL with definitions of the geometrical parameters of the etched ring; b) top view of the ring (red) and tunnel junction (blue).

The optical confinement is realised by the ring (Fig. 1b) etched at the interface between the cavity and the top DBR Such a configuration is possible due to a wafer bounding technique giving access to the cavity since before fusing the DBRs to the active region, the cavity is exposed and permits surface patterning [7]. The etched patterns are concentric with the tunnel junction mesa. In the simulations we vary the etching depth $(L)$ of the pattern, its inner diameter $\left(d_{\mathrm{p}}\right)$ and the width $(w)$. The emitted wavelength of VCSEL, close to the threshold, is about 1310nm. To simulate rigorously the physical phenomena taking place in the device, we use a multiphysical model [8-9] which comprises three-dimensional models of optical (Plane Wave Admittance Method), thermal, electrical and diffusion phenomena (all three based on Finite Element Method). Such a comprehensive model allows to determine precisely the modes modifications driven by different working conditions. We would like to stress that analysis performed according to the usual optical effective models would not explain the behaviour described here, since the refractive indices of particular parts of the device are averaged and 
transformed into uniform blocks defined by constant effective refractive indices along the axis of the device. In such models, the influence of the ring is manifested by a waveguide effect only, omitting losses from leakage and scattering. Our model has been validated by comparison with other numerical approaches for VCSEL optical modelling [10] and shows very good agreement compared to independent measurements [11].

The material parameters used in the analysis are shown in Tab. 1.

Table 1. Physical parameters used in the simulation at $300 \mathrm{~K}$ (* measured value, $* *$ - the value has been found from the comparison of the model with experiment).

\begin{tabular}{|c|c|c|c|}
\hline Material & $\begin{array}{c}\text { Thermal } \\
\text { conductivity } \\
{[\mathrm{W} /(\mathbf{m K})]}\end{array}$ & $\begin{array}{c}\text { Refractive } \\
\text { index at } \\
1.31 \mu \mathrm{m}\end{array}$ & $\mathrm{d} n / \mathrm{d} T[1 / \mathrm{K}]$ \\
\hline $\mathrm{InP}$ & $71[12]$ & $3.21 *$ & $2.7910^{-4} * *$ \\
\hline $\begin{array}{l}\mathrm{QW} \\
\mathrm{In}_{0.67} \mathrm{Al}_{0.2} \mathrm{Ga}_{0.13} \mathrm{As}\end{array}$ & $4[13]$ & $3.6 *$ & $310^{-4} * *$ \\
\hline $\begin{array}{l}\text { Barier and TJ: } \\
\mathrm{In}_{0.42} \mathrm{Al}_{0.32} \mathrm{Ga}_{0.26} \mathrm{As}\end{array}$ & $4[13]$ & $3.5 *$ & $310^{-4} * *$ \\
\hline $\mathrm{Al}_{0.9} \mathrm{Ga}_{0.1} \mathrm{As}$ & $26.3[14]$ & $2.98 *$ & $1.3710^{-4}[15]$ \\
\hline GaAs & $45.4[14]$ & $3.408 *$ & $2.6710^{-4}[15]$ \\
\hline InGaAsP & 19 [16] & $3.5 *$ & $310^{-4} * *$ \\
\hline In & $81.63[17]$ & \multirow[t]{2}{*}{-} & \multirow[t]{2}{*}{-} \\
\hline $\mathrm{Cu}$ & 400.8 [18] & & \\
\hline
\end{tabular}

The optical confinement is realised by two ways of patterning:

- the tunnel junction etched and overgrown with InP [19]

- the air gaps etched at the interface between cavity and DBR.

The contrast between the refractive index of the TJ InAlGaAs layer and the neighbouring InP layer is 0.2 . The influence of the refractive index contrast on the lateral mode distribution is significantly reduced by placing the TJ layer in the node of the standing wave. Air gap patterning, which is introduced at the interface between the cavity and the top DBR is responsible for further stabilization of the mode distribution, rendering it independent of thermal focusing [20]. The interface between the cavity and the DBR is placed in the node position. However, depending on the etching depth, the air gap can coincide with the antinode position and strongly interacts with the mode. The lateral refractive index contrast between air and InP is larger than 2, hence the air gap dominates the confinement of TJ and to some extent makes the mode distribution independent of thermal focusing.

The structure of the VCSEL with a patterned TJ reveals the record power of single mode operation [21]. The size of the optical aperture is the element which limits the power in the single mode regime. The optimal size of the aperture is the effect of the trade-off between small emitted power and multimodal operation. The purpose of the additional air-gap patterning is to introduce the penalty for higher order modes to allow a larger injection in the case of relatively broad apertures and single mode regime.

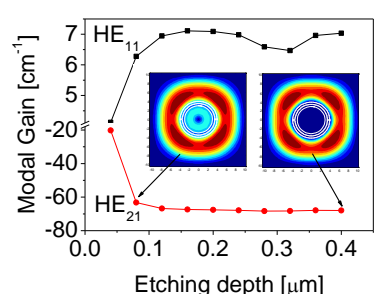

a)

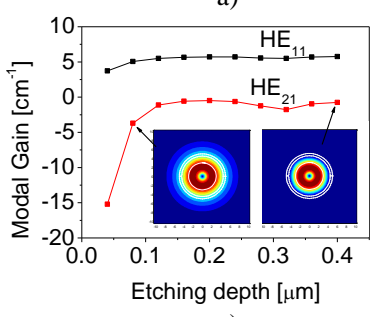

c)

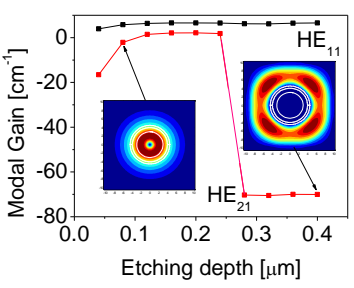

b)
Fig. 2. The modal gain of $\mathrm{HE}_{11}$ and $\mathrm{HE}_{21}$ as the function of the etching depth of the ring for $d_{\mathrm{p}}=7 \mathrm{~mm}, d_{\mathrm{TJ}}=6 \mathrm{~mm}$ and for three different widths: a) $w=0.5 \mu \mathrm{m}$, b) $w=1.0 \mu \mathrm{m}$, a) $w=1.5 \mu \mathrm{m}$.

Figure 2 illustrates the modifications of $\mathrm{HE}_{21}$ mode distribution driven by different parameters of the ring. Figures 2a-c correspond to the rings of the same inner diameter $\left(d_{\mathrm{p}}\right)$ but different widths $(w)$. Larger $w$ enhances the optical confinement which contributes to a larger number of modes confined in the aperture. Figure $2 \mathrm{a}$ shows the impact of the narrowest analysed ring. The increase in the etching depth up to $\sim 0.1 \mu \mathrm{m}$ considerably increases both the modal gain of $\mathrm{HE}_{11}$ and the modal gain difference between $\mathrm{HE}_{11}$ and $\mathrm{HE}_{21}$. The ring pattern confines the fundamental mode and enhances the losses of first order mode since a narrow ring does not confine $\mathrm{HE}_{21}$ mode, which becomes pushed out of the aperture. The etching depth deeper than $\sim 0.1 \mu \mathrm{m}$ does not affect significantly the modal gain difference between the modes, since the etching depth equal to $0.16 \mu \mathrm{m}$ relates to the bottom of the ring being in the anti-node position of the standing wave. Such a configuration relates to the strongest interaction between the etching structure and the mode [22]. Figure 2c illustrates the opposite behaviour which is imposed by a larger width of the ring $(w)$. Both modes become strongly confined by the increase of the etching depth. Figure $2 b$ refers to the transitional behaviour between both described cases. The increase of the etching depth confines $\mathrm{HE}_{21}$ mode initially. If the etching depth becomes deeper $(0.25 \mu \mathrm{m}$ in that particular case) one can observe an abrupt transition in the modal gain of $\mathrm{HE}_{21}$ mode. The transition is the result of the escape of the $\mathrm{HE}_{21}$ mode out of the aperture. The $\mathrm{HE}_{21}$ mode transition might be explained by scattering losses 
which increase with the deepening of the ring. Once they dominate the waveguide effect, the $\mathrm{HE}_{21}$ mode becomes pushed out of the aperture. Such behaviour is not observed in any other method of optical confinement based on the waveguide mechanism. A similar effect of higher order modes discrimination is observed in antiresonant VCSELs but the fundamental mode is burdened with the lateral leakage losses contributing to an increase in the threshold current.

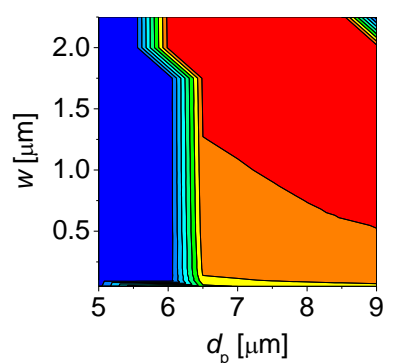

a)

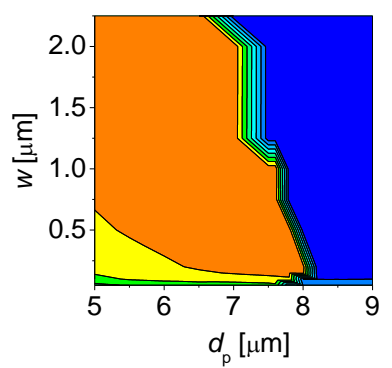

c)

Fig. 3. The modal gain difference between $\mathrm{HE}_{11}$ and $\mathrm{HE}_{21}$ as function of the aperture $\left(d_{\mathrm{p}}\right)$ and width of the ring $(w)$ for $d_{\mathrm{TJ}}=6 \mu \mathrm{m}$ and different injected currents: a) $6 \mathrm{~mA}$, b) $9 \mathrm{~mA}$, c) $12 \mathrm{~mA}$.

Figure 3 collects the maps of the modal gain difference between $\mathrm{HE}_{11}$ and $\mathrm{HE}_{21}$ calculated for different injected currents ranging from 6 to $12 \mathrm{~mA}$. The maps are plotted in the domain of the width of the ring $(w)$ and inner aperture of the ring $\left(d_{\mathrm{p}}\right)$, the depth of the etching is $200 \mathrm{~nm}$ and the tunnel junction aperture $\left(d_{\mathrm{TJ}}\right)$ equals $6 \mu \mathrm{m}$. The yellow color in the maps corresponds to very strong modes discrimination $\left(60 \mathrm{~cm}^{-1}\right)$ which relates to pushing the $\mathrm{HE}_{21}$ mode out of the aperture. Figure 3 revels that the yellowred regions of very strong discrimination are independent of the current injection in the range of $d_{\mathrm{p}}$ from 6 to $8 \mu \mathrm{m}$ and $w<1 \mu \mathrm{m}$. Such a ring design provides stable single mode operation and opens up the possibilities of increasing the maximal emitted power and assures broad wavelength tuning in the single mode regime.

In summary, we demonstrate the results of numerical simulations of $1.3 \mu \mathrm{m}$ InAlGaAs/InP VCSEL with a ring pattern etched at the interface between the cavity and top DBR. The design reveals the unique attribute of selective confinement of the fundamental mode and strong discrimination of higher order modes. The proposed structure allows operation in a broad range of injected currents eliminating the modes competition, which facilitates the improvement of maximal emitted power and increase of the wavelength tuning range in the single mode regime.

This work was supported by the Polish National Science Centre, project DEC-2011/03/N/ST7/03343, project: "A Novel Photonic Crystal Surface Emitting Laser Incorporating a High-Index-Contrast Grating" from the Polish National Centre for Research and Development. M. Dems and J. Walczak acknowledge the support of the Polish National Centre for Research and Development within the project LIDER.

\section{References}

[1] C. Jung, R. Jäger, M. Grabherr, P. Schnitzer, R. Michalzik, B. Weigl, S. Müller, K.J. Ebeling, Electron. Lett. 33, 1790 (1997).

[2] A.-S. Gadallah, R. Michalzik, IEEE Photon. Technol. Lett. 23, 1040 (2011).

[3] D. Zhou, L.J. Mawst, IEEE J. Quantum Elect. 38, 1599 (2002).

[4] E. Kapon, A. Sirbu, Nat. Phot. 3, 27 (2009).

[5] A.J. Danner, J.J. Raftery, T. Kim, P.O. Leisher, A.V. Giannopoulos, K.D. Choquette, IEICE Trans. Electron. E88-C, 944 (2005).

[6] N. Volet, T. Czyszanowski, J. Walczak, L. Mutter, B. Dwir, Z. Micković, P. Gallo, V. Iakovlev, A. Sirbu, A. Caliman, A. Mereuta, E. Kapon Proc. SPIE 8639, 86390S (2013).

[7] A. Sirbu, V. Iakovelv, A. Mereuta, A. Caliman, G. Suruceanu, E. Kapon, Semicond. Sci. Technol. 26, 014016 (2011).

[8] M. Dems, R. Kotynski, K. Panajotov, Opt. Exp. 13, 3196 (2005).

[9] R.P. Sarzala, W. Nakwaski, J. Phys., Condens. Matter 16, S3121 (2004).

[10] D. Xu, C. Tong, S.F. Yoon, W. Fan, D.H. Zhang, M. Wasiak, Ł. Piskorski, K. Gutowski, R.P. Sarzała, W. Nakwaski, Semicond. Sci. Technol. 24, 055003 (2009).

[11] T. Czyszanowski, M. Dems, R.P. Sarzała, K. Panajotov, K.D. Choquette IEEE J. Select. Top. Quant. Electron. 19, 1701908 (2013).

[12] W. Nakwaski, J. Appl. Phys. 64, 159 (1989).

[13] J. Piprek, J. K.White, A.J. SpringThorpe, IEEE J. Quantum Electron. 38, 1253 (2002).

[14] S. Adachi, J. Appl. Phys. 58, R1 (1985).

[15] S. Gehrsitz, F.K. Reinhart, C. Gourgon, N. Herres, A. Vonlanthen, H. Sigga, J. Appl. Phys. 87, 7825 (2000).

[16] S. Adachi, Physical Properties of III-V Semiconductor Compounds (New York, NY, USA, Wiley 1992).

[17] R.J. Sladek, Phys. Rev. 97, 902 (1955).

[18] N.V. Zavaritskii, A.G. Zeldovich, Zh. Tekh. Fiz. 26, 2032 (1956).

[19] L. Mutter, V. Iakovlev, A. Caliman, A. Mereuta, A. Sirbu, E. Kapon, Opt. Exp. 17, 8558 (2009).

[20] L. Mutter, B. Dwir, A. Caliman, V. Iakovlev, A. Mereuta, A. Sirbu, E. Kapon, Opt. Exp. 19, 4827 (2011).

[21] E. Kapon, A. Sirbu, Nat. Phot. 3, 27 (2009)

[22] T. Czyszanowski, M. Dems, R.P. Sarzała, W. Nakwaski, K. Panajotov, IEEE J. Quantum Electron 47, 1291 (2011). 